\title{
Empirically adapted or personalized antibiotic prophylaxis in select cranial neurosurgery?
}

\author{
Thomas Mindermann ${ }^{1}$ (D) \\ Received: 26 August 2020 / Accepted: 27 August 2020 / Published online: 2 September 2020 \\ (C) Springer-Verlag GmbH Austria, part of Springer Nature 2020
}

\section{Introduction}

The development of a safe and effective antibiotic prophylaxis in surgery has been developing since the 1970s. A few groundbreaking publications have led to today's concept of single-dose or ultra-short antibiotic prophylaxis in most surgical interventions including cranial neurosurgery. Over the decades, a few basic rules of antibiotic prophylaxis have evolved such as the avoidance of antibiotics which are first choice therapeutic agents, their application before skin incision, the appropriate selection of antibiotic agents effective against the expected pathogens, and the application of a single-dose or an ultra-short prophylaxis rather than extended regimes. By following these rules, the risk of the emergence of resistant strains is reduced to a minimum while providing a good safeguard against postoperative infections without spoiling potential candidates for an antibiotic therapy in the case of infection. Lately, several publications have surfaced dealing with the question which particular prophylactic regime is adequate and if antibiotic prophylaxis in cranial neurosurgery should be individually tailored or not $[1,2,7]$. Is it time to abandon empirically adapted and proven regimes of antibiotic prophylaxis for personalized regimes in endonasal skull base surgery and other selected cranial interventions?

\section{Historical context}

In this context, it is worthwhile to recollect briefly the historically most important publications for surgery and neurosur-

This article is part of the Topical Collection on Pituitaries

Thomas Mindermann tmindermann@hin.ch

$1 \quad$ Klinik Im Park, Zurich, Switzerland gery addressing antibiotic prophylaxis. In the decade from 1970 until 1980, Cruse and Foord introduced and established the concept of four different surgical categories with distinctly different risks of postoperative wound infection in the absence of antibiotic prophylaxis: (i) "clean" with a $1.5 \%$ risk, (ii) "clean-contaminated" with a $7.7 \%$ risk, (iii) "contaminated" with a $15.2 \%$ risk, and (iiii) "dirty" with a $40 \%$ risk of infection [4]. It became increasingly clear that about $80 \%$ of wound infections are caused by the patient's own saprophytes of the skin or the mucosa, that the ideal timing for antibiotic prophylaxis is its application before skin incision, and that for obvious reasons no first-line therapeutics should be used for prophylaxis. In 1972, it was a prospective, controlled, and double-blind study in cardiac surgery which favored and established single-dose antibiotic prophylaxis over multipledose regimes in surgery of the "clean" category [3]. In 1985, a study published a wide risk of infections following clean cranial neurosurgery in the absence of antibiotic prophylaxis [8]. The authors showed that re-operations for gliomas had an $11 \%$ risk of deep wound infection, while it was only $2.5 \%$ for regular craniotomies. Therefore and considering the scalp's microbiome, cranial neurosurgery though officially categorized as "clean" should probably rather be categorized as "clean-contaminated" or even "contaminated" in some instances due to its wound infection rates reflecting the scalp's dense colonization with commensals. In the 1980s, singledose antibiotic prophylaxis has been established not only for "clean" but also for "clean-contaminated" and "contaminated" procedures in the various fields of surgery [5]. Antibiotic prophylaxis has then been recommended for cranial neurosurgery not only because of wound infection rates paralleling those of "clean-contaminated" procedures but also because of what is at stake in the case of postoperative infections. Today, we see a renewed interest in single-dose or ultra-short antibiotic prophylaxis in cranial neurosurgery raising the issue of a tailored antibiotic prophylaxis. Individually tailored or more accurately personalized antibiotic prophylaxis seems to be seen as the answer to issues related to the increased use of access 
pathways through the nostrils and the paranasal sinuses to skull base lesions or to site infections following cranioplasty $[2,7]$.

\section{Cranial neurosurgery}

The scalp's microbiome seems to match that of the axilla or the pubes in terms of density. This and the fact that the vast majority of postoperative wound infections derive from bacteria colonizing the patients' skin requires an antibiotic prophylaxis covering for Gram-positive bacteria in most cranial interventions. Several agents proved to be effective in craniotomies and in shunt surgery, yet not all of them cover for postoperative infections with methicillin-resistant Staphylococcus aureus (MRSA), and some of them are considered first-line agents for an antibiotic therapy in the case of an infection with MRSA [1]. An empirically adapted antibiotic prophylaxis established by research data has been in use for a long time for most of cranial neurosurgery. Nowadays, more and more skull base interventions are done through endonasal access pathways which require a different and adequately adapted antibiotic prophylaxis because of the nasal mucosa's and the paranasal sinuses' pathogens. This in turn has led to two divergent strategies in antibiotic prophylaxis: either an empirically adapted antibiotic prophylaxis with the same regime for all patients or an individually tailored or personalized antibiotic prophylaxis with different regimes for each patient. Yet, so far a proper distinction between those two concepts has not been made.

\section{Tailored antibiotic prophylaxis}

Should a single-dose or ultra-short antibiotic prophylaxis be tailored or not? The short answer is yes since the antibiotic should certainly be adapted to cover for the pathogens to be expected in case of a postoperative infection such as Grampositive bacteria in cranial neurosurgery. As long as the antibiotic is empirically well adapted and effective against the expected pathogens, it does not seem to matter much which particular one is chosen [1]. The adequate choice among the viable candidates has more to do with the other rules of antibiotic prophylaxis such as the avoidance of first-line therapeutics. A prospective, randomized, placebo-controlled study addressed the prophylactic coverage of Gram-positive bacteria including MRSA in craniotomies and shunt surgery avoiding the application of first choice therapeutic agents such as vancomycin [6]. This study's single-dose antibiotic prophylaxis with $500 \mathrm{mg}$ fusidic acid proved to be safe and effective in cranial neurosurgery excluding that engaging transnasal pathways which was at the time not part of the study. Such an antibiotic prophylaxis seems to be fit for all patients undergoing cranial neurosurgery with the exception of endonasal procedures. Do endonasal procedures or other procedures with a high infection rate justify a deviation from a generally adapted or tailored prophylaxis fitting all patients towards a personalized prophylaxis adapted to an individual patient's mucosal or skin colonization? This question needs to be addressed in more detail.

\section{Access pathways through the nares and the paranasal sinuses}

Per definition, all endonasal procedures belong to the "cleancontaminated" risk category since the surgical pathway is accessed through the nasal and paranasal sinus' mucosa. This is the main reason why the choice of antibiotic prophylaxis in transnasal neurosurgery may differ from that of other cranial interventions. A different spectrum of potential pathogens is to be expected and requires an adapted antibiotic prophylaxis. In standard cranial surgery, it is sufficient for an antibiotic prophylaxis to cover for Gram-positive bacteria including MRSA. In endoscopic endonasal skull base surgery (EESBS), the presence of Gram-positive or Gram-negative bacteria, MRSA, or anaerobe bacteria in the nasal cavity and the paranasal sinuses must be considered when choosing an adapted antibiotic prophylaxis. Yet, is there room for a personalized antibiotic prophylaxis according to preoperative nasal or rectal swabs on a routine basis rather than an empirically adapted prophylaxis? While a study from 2020 seems to favor such an approach, it fails to prove a benefit of such a personalized prophylaxis [2]. While such a prophylactic regime seems to be safe and effective, it is cumbersome, and in the case of that study, it has the disadvantage of employing vancomycin which serves as a first choice therapeutic in infections with MRSA. Theoretically, an adapted prophylaxis based on empirical data with a single-dose or ultra-short antibiotic prophylaxis with fusidic acid plus metronidazole should provide an equally effective safeguard against the vast majority of pathogens to be expected in EESBS including MRSA.

\section{Cranioplasty}

Cranioplasty with or without autologous material is another intervention with notoriously high infection rates which came to the attention of those favoring a personalized rather than empirically adapted antibiotic prophylaxis. A study from 2020 addresses the issue of a personalized antibiotic prophylaxis taking preoperative swabs from the scalp and employing vancomycin in the presence of MRSA [7]. Once again, this personalized prophylaxis led to a significant reduction in postoperative site infections, but the routine prophylaxis with cefazolin to which the personalized prophylaxis was 
compared was probably from an empirical point of view not an ideal choice. Also in this case, an empirically adapted prophylaxis with fusidic acid for all patients may have solved the problem by covering for the expected Gram-positive pathogens including MRSA and at the same time avoiding the use of vancomycin which may serve as a first-line therapeutic.

\section{Conclusion}

A personalized antibiotic prophylaxis guided by preoperative swabs is certainly effective in EESBS and cranioplasty, but is it really necessary? The application of an adapted antibiotic prophylaxis to all patients undergoing the same intervention guided by empirical data covering for the expected pathogens and avoiding potential first-line therapeutics may possibly be the better choice. Such a regime has the advantage of eliminating the risk of wrongly applied antibiotics to individual patients undergoing the same intervention in an everyday clinical setting. It would favor the application of (i) fusidic acid in cranial neurosurgery in general including cranioplasty and (ii) fusidic acid plus metronidazole in cranial neurosurgery involving nasal and paranasal access pathways. Any future study on personalized antibiotic prophylaxis guided by preoperative swabs should include a study arm with an adequately chosen, empirically adapted regime, e.g., of this kind which probably fits the vast majority of patients rather than including study arms of less appropriately selected regimes as it has been done recently.

\section{References}

1. Cao Y, Wang B, Shan J, Gong Z, Kuang J, Gao Y (2020) Indirect comparison of efficacy between different antibiotic prophylaxis against the intracranial infection after craniotomy. Antimicrob Resist Infect Control 9:22. https://doi.org/10.1186/s13756-02000784-9

2. Ceraudo M, Prior A, Balestrino A, Anania P, Camera M, Fiaschi P, Gatto F, Riccardi N, Zona G, Criminelli Rossi D (2020) Ultra-short single-agent antibiotic prophylaxis guided by preoperative microbiological nasal swabs in endoscopic endonasal skull base surgery. Acta Neurochir

3. Conte JE, Cohen SN, Roe BB, Elashoff RM (1972) Antibiotic prophylaxis and cardiac surgery. A prospective double-blind comparison of single-dose versus multiple-dose regimes. Ann Int Med 76: 943-949

4. Cruse PJE, Foord RRN (1980) The epidemiology of wound infection. A 10-year prospective study of 62'939 wounds. Surg Clin N Am 60:27-40

5. Mindermann T, Gruber UF (1985) Indications for single-dose antibiotic prophylaxis in surgery and gynecology. Hans Huber Publishers, Toronto $56 \mathrm{pp}$

6. Mindermann T, Zimmerli W, Gratzl O (1993) Randomized placebocontrolled trial of single-dose antibiotic prophylaxis with fusidic acid in neurosurgery. Acta Neurochir 121:9-11

7. Paredes I, Lagares A, San-Juan R, Castano-Leon AM, Gomez P-A, Jimenez-Roldan L, Panero I, Eiriz C, Garcia-Perez D, Moreno LM (2020) Reduction of the infection rate of cranioplasty with a tailored antibiotic prophylaxis: a nonrandomized study. Acta Neurochir. https://doi.org/10.1007/s00701-020-04508-1

8. Tenney JH, Vlahov D, Salcman M, Ducker TB (1985) Wide variation in risk of wound infection following clean neurosurgery. $\mathrm{J}$ Neurosurg 62:243-247

Publisher's note Springer Nature remains neutral with regard to jurisdictional claims in published maps and institutional affiliations. 